Research Article

\title{
Neutrophil-Lymphocyte Ratio as a Prognostic Parameter in NSCLC Patients Receiving EGFR-TKIs: A Systematic Review and Meta-Analysis
}

\author{
Mingbo Tang $\mathbb{C}^{1},{ }^{1}$ Xinliang Gao $\mathbb{D},{ }^{1}$ He Sun $\mathbb{D}^{1},{ }^{1}$ Suyan Tian $\mathbb{D}^{2},{ }^{2}$ Junxue Dong $\mathbb{D},{ }^{3}$ \\ Zhao Liu $(\mathbb{D}){ }^{1}$ and Wei Liu $(\mathbb{D})^{1}$ \\ ${ }^{1}$ Department of Thoracic Surgery, The First Hospital of Jilin University, Changchun 130021, Jilin, China \\ ${ }^{2}$ Department of Division of Clinical Research, The First Hospital of Jilin University, Changchun 130021, Jilin, China \\ ${ }^{3}$ Department of Molecular Biology, Max Planck Institute for Infection Biology, Berlin 10117, Germany
}

Correspondence should be addressed to Wei Liu; weiliu971@gmail.com

Received 18 December 2020; Revised 5 January 2021; Accepted 7 January 2021; Published 20 January 2021

Academic Editor: Nicola Silvestris

Copyright (C) 2021 Mingbo Tang et al. This is an open access article distributed under the Creative Commons Attribution License, which permits unrestricted use, distribution, and reproduction in any medium, provided the original work is properly cited.

Objective. To research the impact of neutrophil-lymphocyte ratio (NLR) as a prognostic parameter in non-small-cell lung cancer (NSCLC) patients treated with epidermal growth factor receptor tyrosine kinase inhibitors (EGFR-TKIs). Methods. We searched the databases such as the American Society of Clinical Oncology (ASCO), EMBASE, PubMed, the European Society of Medical Oncology (ESMO), Wanfang, and CNKI for articles illustrating the impact of pretreatment NLR on survival data in NSCLC patients undergoing EGFR-TKIs treatment. We did a meta-analysis for overall survival (OS) and progression-free survival (PFS). Results. We recruited 10 studies in our meta-analysis. Our study suggested that patients with low NLR had better PFS (hazard ratio $(\mathrm{HR})=1.67,95 \%$ confidence interval $(\mathrm{CI})=(1.16-2.39)$, and $P$ value $=0.005)$ and $\mathrm{OS}(\mathrm{HR}=1.66,95 \%$ $\mathrm{CI}=(1.08-2.55)$, and $P$ value $=0.02)$ in comparison to patients with high NLR. Conclusion. In conclusion, our meta-analysis revealed that lower NLR predicted a better survival (PFS and OS) in patients receiving the treatment of EGFR-TKIs.

\section{Background}

Lung cancer is among the leading causes of cancer death among both genders, with one-quarter of cancer death due to lung cancer [1]. Non-small-lung cancer (NSCLC) takes about $85 \%$ of all lung cancer types. Over $60 \%$ of patients with NSCLC had an expression of epidermal growth factor receptor (EGFR) [2]. Thus, EGFR has been a very vital treatment target for these NSCLC patients, which is more often detected in females and nonsmokers [3]. Tyrosine kinase inhibitors (TKIs) are important treatment targets for patients harboring activating mutations in the tyrosine kinase domains of the EGFR gene. Many studies identified the prognostic biomarkers for NSCLC patients undergoing EGFR-TKIs treatment, and neutrophil-to-lymphocyte ratio (NLR) has been an interesting field. The NLR is calculated with absolute neutrophil counts divided by the absolute lymphocyte counts of a full blood count, and thus, the test cost of this biomarker is not expensive. NLR can be used as an inflammatory biomarker that indicates systematic inflammation [4]. Inflammation has a fundamental function in the tumor development and progress of cancer cells including proliferation, angiogenesis, and metastasis. Thus, NLR could serve as a prognostic factor. An Italian study consisting of 63 end-stage NSCLC patients with EGFR mutation treated with EGFR-TKIs suggested that patients with NLR lower than 3.5 had longer progression-free survival (PFS) and overall survival (OS) in comparison with those with NLR higher than 3.5 (PFS: hazard ratio $(\mathrm{HR})=$ 2.275, $P$ value $=0.007 ;$ OS: $\mathrm{HR}=2.699, P$ value $=0.018)[5]$. Another retrospective Japanese study recruiting 205 stage IV NSCLC patients under EGFR-TKIs treatment suggested that patients with NLR lower than 3.55 had longer PFS and OS compared with those with NLR higher than 3.55 (for PFS: 
$\mathrm{HR}=1.82, P$ value $<0.0001$; for $\mathrm{OS}: \mathrm{HR}=1.78, P$ value $<0.001$ ) [6]. However, studies did not reach consistency $[7,8]$.

The previous meta-analysis revealed that NLR predicted elevated length of survival in NSCLC patients with systematic treatment including chemotherapy, targeted therapy, and immunotherapy $[9,10]$. However, recruiting clinical studies with different treatment methods and patients in different stages increased the heterogeneity of our study. No meta-analysis focused on the impact of NLR on prognosis in NSCLC patients receiving EGFR-TKIs. Thus, we decided to do a meta-analysis investigating clinical studies about pretreatment NLR level on survival in NSCLC patients with EGFR-TKIs treatment. Our hypothesis is that patients with lower NLR could be a prognostic parameter for improved length of survival in patients with NSCLC treated with EGFR-TKIs.

\section{Methods}

2.1. Literature Search. The American Society of Clinical Oncology (ASCO), EMBASE, PubMed, European Society of Medical Oncology (ESMO), Wanfang, and CNKI databases were searched by independent researchers with the following keywords: non-small cell lung cancer, lung cancer, neutrophil lymphocyte ratio, epidermal growth factor receptor tyrosine kinase inhibitors, NSCLC, NLR, EGFR-TKIs, erlotinib, gefitinib, icotinib, afatinib, osimertinib, survival, PFS, and OS. We followed the methods of Xu et al. [11]. Two researchers searched the database for all published papers.

2.2. Inclusion Criteria. We selected published articles meeting all the following criteria: (1) clinical trials of patients who were cytologically or pathologically diagnosed with NSCLC and received EGFR-TKIs; (2) the clinical trials evaluated the length of survival data, including PFS and OS with a HR and also 95\% confidence interval (CI).

2.3. Extraction of Study Results. Two independent medical doctors in our department read and approved all the papers independently and finally reached a consensus about the inclusion of the studies. When they cannot reach a consensus, a third researcher took part in the study inclusion procedures. We used the criteria defined by Cochrane Handbook for Systematic Reviews of Interventions version 5.1.0 [12], which is commonly used for meta-analysis. We summarized the study characteristics including the name of the first author, the publication time, country information, number of patients, study design, NLR cutoff values, treatment, median PFS, median OS, and follow-up time (Table 1).

2.4. Meta-Analysis. PFS and OS were chosen as the primary endpoints of our systematic meta-analysis. The PFS and OS correlated with NLR are summarized in Table 1 . We calculated HR with 95\% CI as indicators of prognosis with Review Manager (RevMan) version 5.4. Publication bias was calculated using Begg's and Egger's tests and funnel plot. We used the chi-square test and the $I^{2}$ statistic to evaluate the statistical heterogeneity. An $I^{2}$ value $>50 \%$ was considered to suggest a heterogeneity of various studies. When significant heterogeneity was detected, a random-effects model was conducted. An $I^{2}$ below 50\% means no significant heterogeneity between these study results, and thus, a fixed-effects model was conducted.

\section{Results}

3.1. Study Characteristics of the Recruited Studies. In total, 10 clinical studies met the inclusion criteria and thus were included in our meta-analysis, with 9 trials about the significant relevance of NLR on PFS and 7 trials about the significant relevance of NLR on OS. The study flow diagram is illustrated in Figure 1. The study characteristics of the ten recruited articles are summarized in Table 1, including author's name, publication year, patient source (country), number of patients, study design, NLR cutoff values, treatment, median PFS, median OS, and follow-up time. All 10 studies met the allocation concealment.

3.2. Meta-Analysis regarding the Prognostic Relevance of NLR on PFS. We recruited 9 clinical trials [5-8, 13-17] including 931 NSCLC patients and investigated the comparison of PFS among patients with low NLR versus patients with high NLR. Our meta-analysis indicated that patients with low NLR had better PFS compared with patients with high NLR $(\mathrm{HR}=1.67,95 \% \mathrm{CI}=(1.16-2.39)$, and $P$ value $=0.005$, Figure 2).

3.3. Meta-Analysis regarding the Prognostic Relevance of NLR on OS. We recruited 7 clinical trials $[5-8,15,17,18]$ including 2055 NSCLC patients and investigated comparison of OS among patients with low NLR versus patients with high NLR. Our meta-analysis indicated that patients with low NLR had better OS compared with patients with high NLR (HR, 1.66, 95\% CI $=(1.08-2.55)$, and $P$ value $=0.02$, Figure 3).

3.4. Publication Bias. No publication bias was detected in our meta-analysis using funnel plot, Egger's test, and Begg's test (all $P$ values $>0.05$ ) .

\section{Discussion}

Our study suggested that NLR could serve as a prognostic factor for PFS and OS in NSCLC patients undergoing EGFR-TKIs treatment. NLR is calculated as the ratio of circulating neutrophil to lymphocyte counts. Neutrophils serve as especially important cells in inflammatory response. Furthermore, neutrophils, the most abundant type of leukocytes in blood accounting for $50-70 \%$ of all leukocytes, with a nearly seven-hour half-life in healthy people [19], specifically support the initiation of metastasis [20]. A study using mouse breast cancer model suggested that neutrophil-derived leukocytes support the 


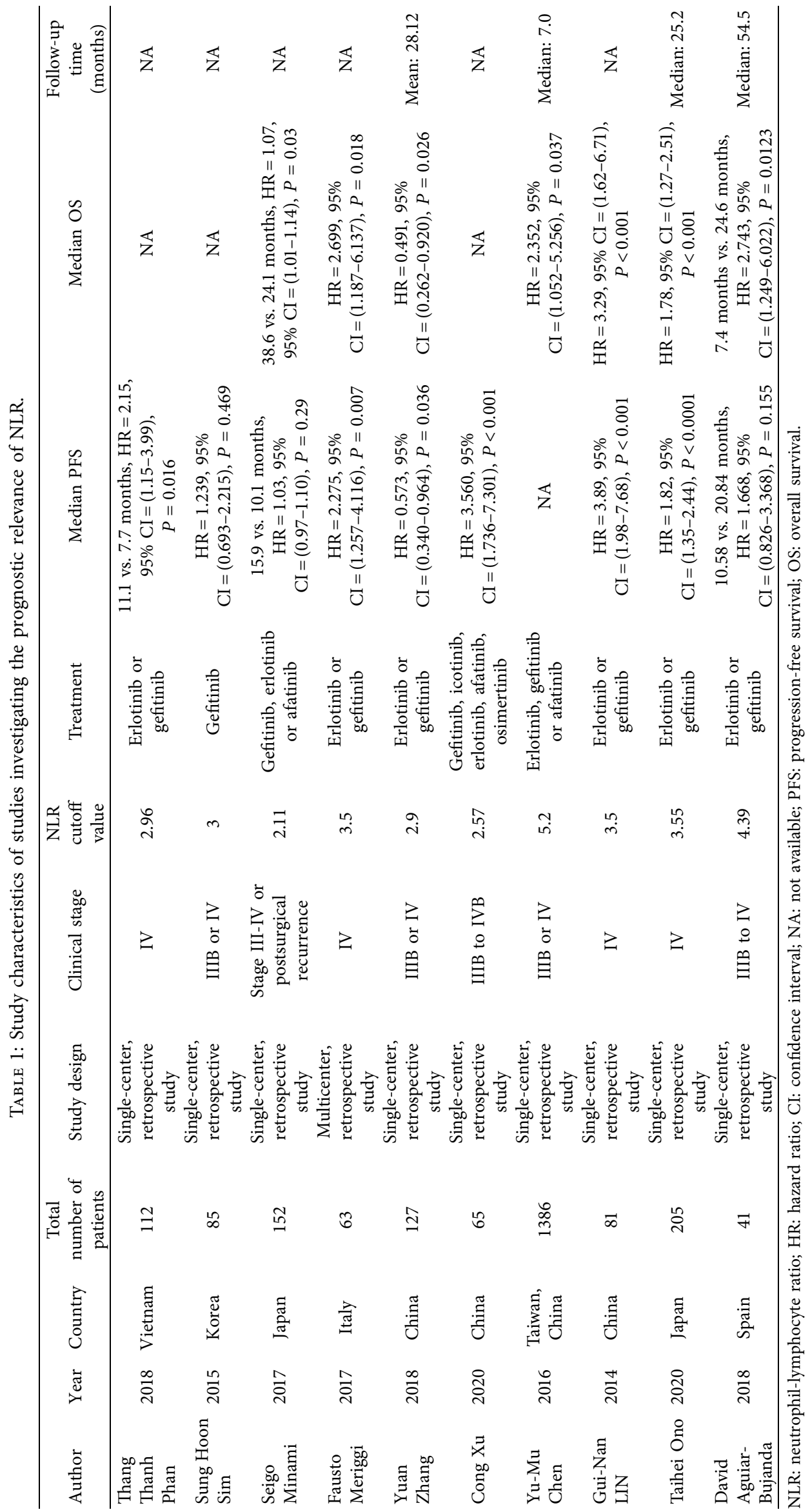




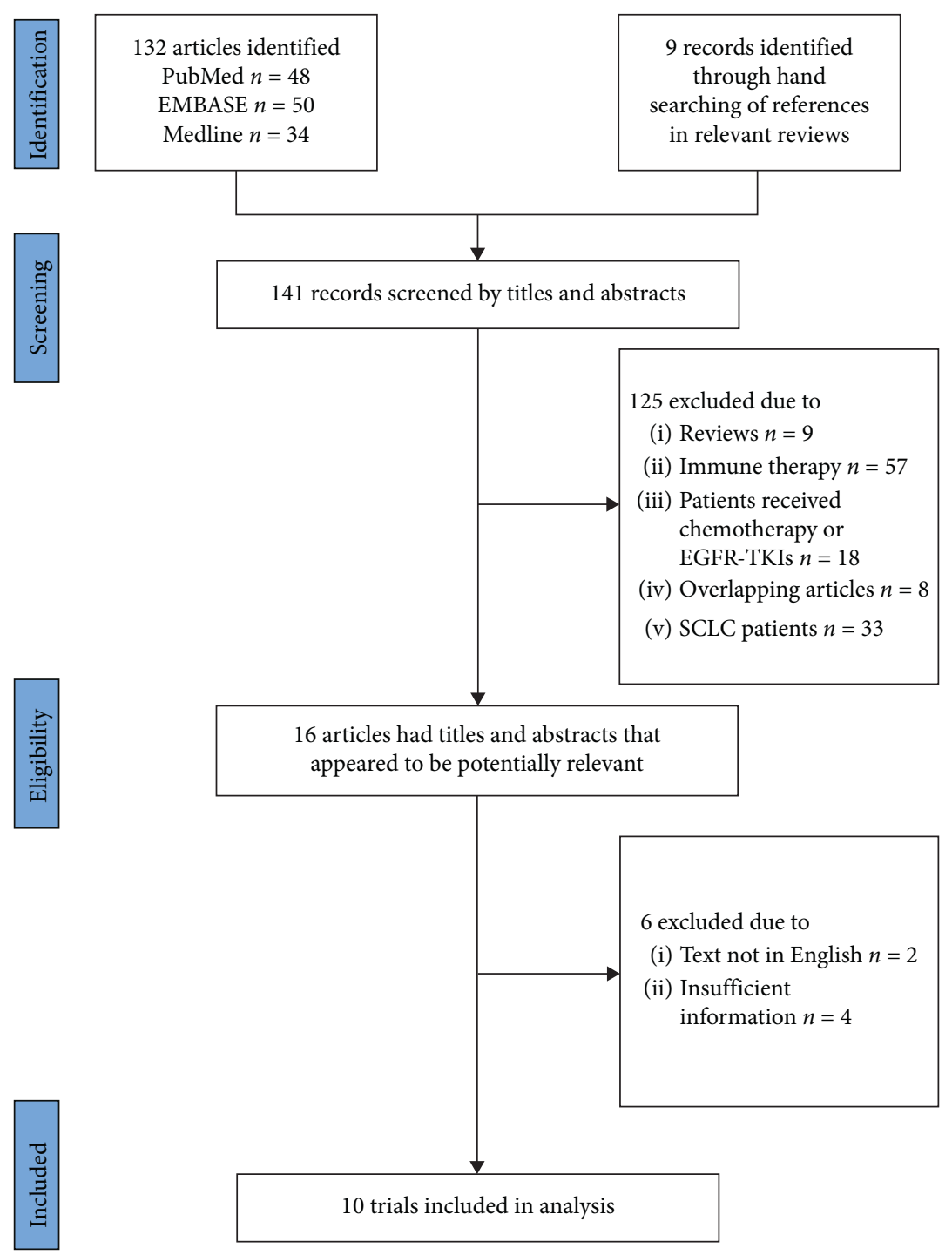

FIgURE 1: Study flow diagram.

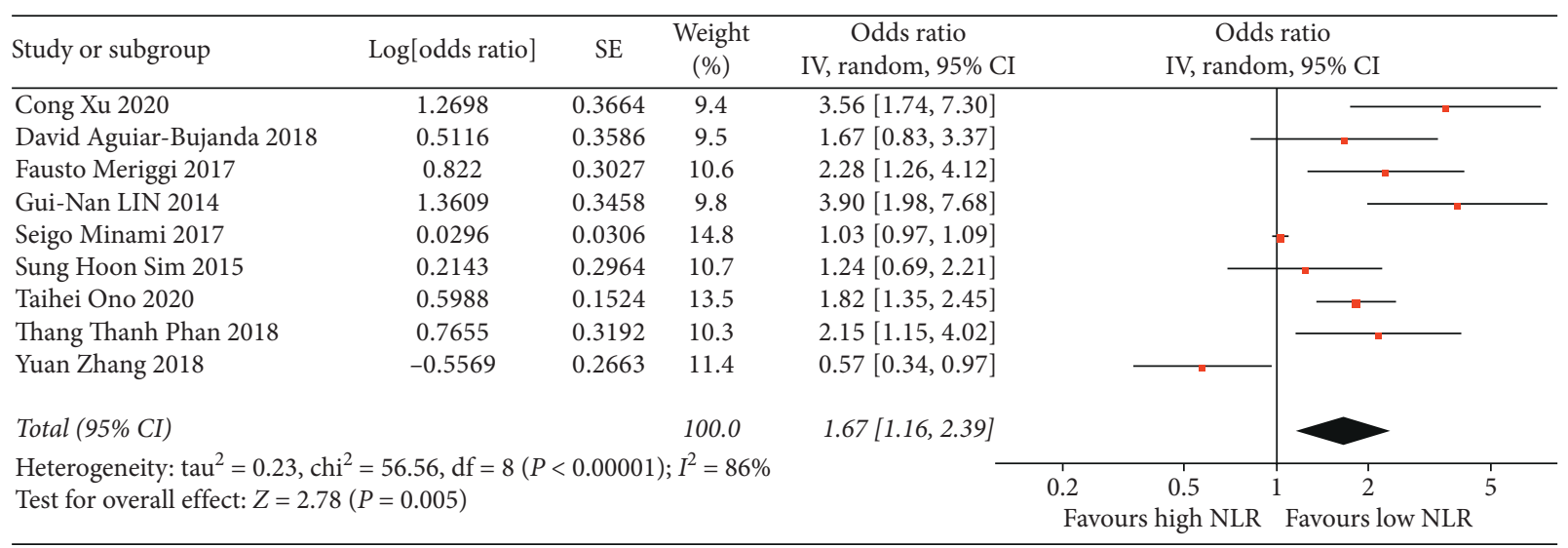

FIgURE 2: Meta-analysis of the impact of NLR on PFS in NSCLC patients with the treatment of EGFR-TKIs.

colonization of cancer cells with a higher tendency of metastasis [20]. Neutrophils expand both in the tumor microenvironment and throughout the body, which in tumor-bearing hosts can oppose or potentiate the progression of cancer cells. These two types of neutrophil behavior are regulated by signaling pathways regulated 


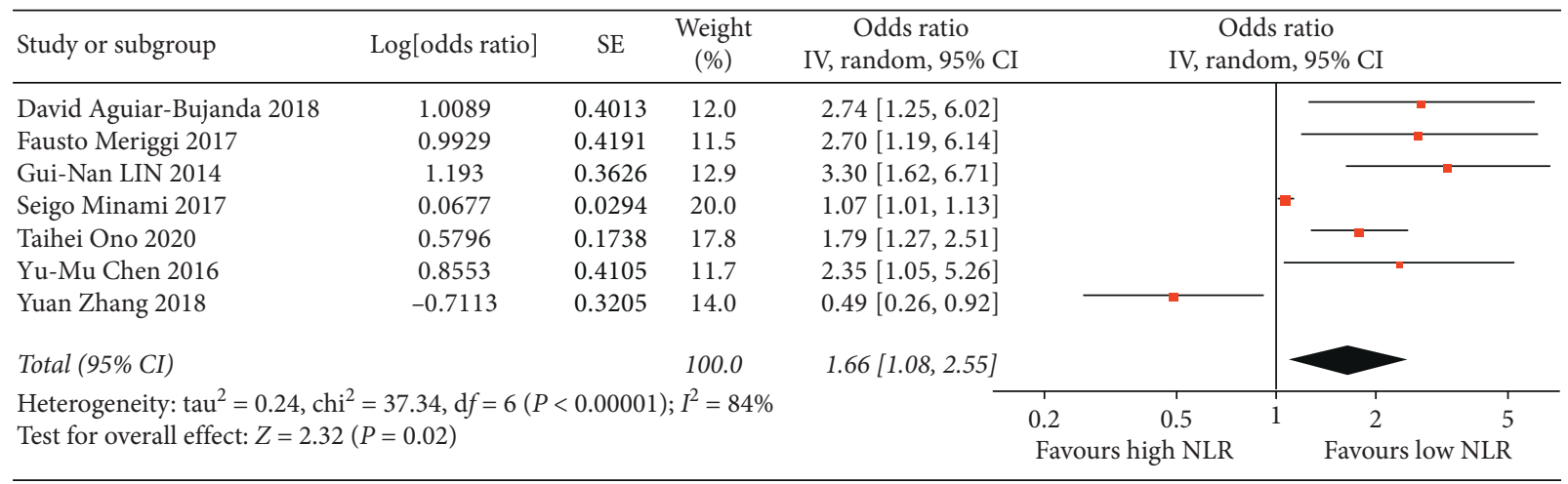

FIGURE 3: Meta-analysis of the impact of NLR on OS in NSCLC patients with the treatment of EGFR-TKIs.

in the tumor microenvironment by tumor cells or stromal cells, which have the function of educating neutrophils to execute the death of the tumor or support tumor spread [21]. Patients with several cancer types, including but not limited to lung cancer, always have a higher number of circulating neutrophils [22]. Animal studies suggested that noncirculating neutrophils are retained longer time in tumor tissues compared to the time in the spleen, indicating that tumor microenvironment encourages the survival of neutrophils [23]. Evidence suggested that circulating neutrophils had a half-life in cancer patients as long as 17 hours [24]. A longer half-life indicated that neutrophils have more time to perform the carcinogenesis during tumor development. The role of neutrophils in tumor development can be regulated by tumor growth factor- $\beta$ (TGF$\beta$ ). A mouse model of subcutaneous mesothelioma tumors treated with a TGF- $\beta$ inhibitor proved that neutrophils support the growth of tumor cells by inhibiting $\mathrm{CD} 8^{+} \mathrm{T}$ cells in the untreated group, while opposed the tumor growth through cytotoxic ability in the TGF$\beta$-treated group. Neutrophils work as a link between inflammation and cancer and have a tumor growthpromoting effect [25]. NLR had a prognostic role in other cancers [26]. In patients with hepatocellular carcinoma (HCC) receiving sorafenib, NLR also showed a prognostic role. Patients with NLR higher than 3 had a lower median PFS compared with those with NLR lower than 3 (2.6 vs. 3.3 months, $P$ value $<0.049$ ), but no significant difference was observed regarding median OS [27]. Our study also supported that patients with low NLR had better survival data in NSCLC patients undergoing EGFR-TKIs treatment, indicating neutrophil as a negative prognostic parameter in cancer patients.

Lymphocyte keeps a vital role in tumor development for mediating anticancer immunity. There are two main types of lymphocytes, B lymphocytes and $\mathrm{T}$ lymphocytes. $\mathrm{T}$ lymphocytes are defined by the expressions of T-cell receptors (TCRs) for recognizing antigens. There are two types of $\mathrm{T}$ cells, which are $\mathrm{CD} 4^{+} \mathrm{T}$ helper cells $\left(\mathrm{T}_{\mathrm{H}}\right)$ and $\mathrm{CD} 8^{+}$ cytotoxic lymphocytes (CTL). CD8 ${ }^{+}$CTLs are critical mediators in the antitumor immunity due to their function to directly kill cancer cells [28]. CD8 ${ }^{+}$CTLs could produce interferon- $\gamma$ (IFN- $\gamma$ ), which could enhance their ability to motility, particularly speed and also its cytotoxic function [29]. Studies have shown a positive association between increased $\mathrm{CD}^{+}$CTLs in the tumor microenvironment and better prognosis in cancer patients, including but not limited to cervical cancer patients, breast cancer patients, and colorectal cancer patients [30-32]. Thus, an elevated $\mathrm{CD}^{+}$ CTLs could link to a better prognosis in many cancer types. $\mathrm{CD} 4^{+} \mathrm{T}$ cells enhance the antitumor immunity by providing help for $\mathrm{CD}^{+}$CTLs and antibody responses, together with the help of secretion of the interferon-gamma (IFN- $\gamma$ ) and tumor necrosis factor- $\alpha$ (TNF- $\alpha$ ) [33]. Our study indicated the same results to previous studies, proving that higher NLR links to better prognosis in NSCLC patients.

Previous studies indicate that, for NSCLC patients receiving immunotherapy, pretreatment NLR serves as a prognostic factor [34]. Our study focused on the NSCLC patients undergoing EGFR-TKIs since more than $60 \%$ of NSCLC patients express the EGFR gene and could be candidates for EGFR-TKIs treatment. It is of great significance to illustrate the prognostic factor for this cohort of patients. Thus, our study was the first to prove the association of lower NLR with better PFS ( $\mathrm{HR}=1.67,95 \%$ $\mathrm{CI}=(1.16-2.39)$, and $P$ value $=0.005)$ and $\mathrm{OS}(\mathrm{HR}=1.66$, $95 \% \mathrm{CI}=(1.08-2.55)$, and $P$ value $=0.02)$ in NSCLC patients treated with EGFR-TKIs.

Our study also has limitations. The studies were recruited using various cutoff points of NLR, ranging from 2.11 to 5.2. The reason might be different studies using the best cutoff point that differentiated the patients that could gain survival benefit. This increased the heterogeneity of our study and raised the difficulty of this biomarker to be used in the clinic. Thus, a large-scale clinical trial is needed to define a cutoff point of the NLR that could be used in the clinic. Nevertheless, our study showed the tendency of worse survival in NSCLC patients with higher pretreatment NLR levels. NLR, as a reasonable and not expensive 
biomarker, could be used as a clinical routine in NSCLC patients.

\section{Conclusion}

In conclusion, our meta-analysis revealed that lower NLR predicted better PFS and OS in NSCLC patients receiving EGFR-TKIs.

\section{Data Availability}

All data generated or analyzed during this study are included in this published article.

\section{Conflicts of Interest}

The authors declare that they have no conflicts of interest.

\section{Authors' Contributions}

Wei Liu designed the study, Mingbo Tang and Xinliang Gao collected the data, He Sun did the data analysis, and Mingbo Tang wrote the first version of the manuscript. All the other authors revised and approved the manuscript.

\section{Acknowledgments}

The authors thank all patients who took part in studies that were recruited in this systematic review and meta-analysis.

\section{References}

[1] R. L. Siegel, K. D. Miller, and A. Jemal, "Cancer statistics, 2019," CA: A Cancer Journal for Clinicians, vol. 69, no. 1, pp. 7-34, 2019.

[2] G. da Cunha Santos, F. A. Shepherd, and M. S. Tsao, "EGFR mutations and lung cancer," Annual Review of Pathology: Mechanisms of Disease, vol. 6, no. 1, pp. 49-69, 2011.

[3] N. Karachaliou, M. Fernandez-Bruno, J. W. P. Bracht, and R. Rosell, "EGFR first- and second-generation TKIs-there is still place for them in EGFR-mutant NSCLC patients," Translational Cancer Research, vol. 8, no. 1, pp. S23-S47, 2018.

[4] E. C. Martins, L. D. F. Silveira, K. Viegas et al., "Neutrophillymphocyte ratio in the early diagnosis of sepsis in an intensive care unit: a case-control study," Revista Brasileira de Terapia Intensiva, vol. 31, no. 1, pp. 64-70, 2019.

[5] F. Meriggi, C. Codignola, G. D. Beretta et al., "Significance of neutrophil-to-lymphocyte ratio in western advanced EGFRmutated non-small cell lung cancer receiving a targeted therapy," Tumori Journal, vol. 103, no. 5, pp. 443-448, 2017.

[6] T. Ono, S. Igawa, S. Kurahayashi et al., "Impact of neutrophilto-lymphocyte ratio in patients with EGFR-mutant NSCLC treated with tyrosine kinase inhibitors," Investigational New Drugs, vol. 38, no. 3, pp. 885-893, 2020.

[7] D. Aguiar-Bujanda, A. Dueñas-Comino, S. Saura-Grau et al., "Neutrophil to lymphocyte ratio as a prognostic factor in European patients with epidermal growth factor receptormutant non-small cell lung cancer treated with tyrosine kinase inhibitors," Oncology Research and Treatment, vol. 41, no. 12, pp. 755-761, 2018.

[8] S. Minami, Y. Ogata, S. Ihara, S. Yamamoto, and K. Komuta, "Neutrophil-to-lymphocyte ratio predicts overall survival of advanced non-small cell lung cancer harboring mutant epidermal growth factor receptor," World Journal of Oncology, vol. 8, no. 6, pp. 180-187, 2017.

[9] Z. Wang, P. Zhan, Y. Lv et al., "Prognostic role of pretreatment neutrophil-to-lymphocyte ratio in non-small cell lung cancer patients treated with systemic therapy: a meta-analysis," Translational Lung Cancer Research, vol. 8, no. 3, pp. 214-226, 2019.

[10] Q. T. Zhao, Y. Yang, S. Xu et al., "Prognostic role of neutrophil to lymphocyte ratio in lung cancers: a meta-analysis including 7054 patients," OncoTargets and Therapy, vol. 8, pp. 27312738, 2015.

[11] Y. Xu, Y. Qiu, S. Yuan, and H. Wang, "Prognostic implication of human papillomavirus types in cervical cancer patients: a systematic review and meta-analysis," Infectious Agents and Cancer, vol. 15, no. 1, p. 66, 2020.

[12] T. J. Chien, C. H. Hsu, C. Y. Liu, and C. J. Fang, "Effect of acupuncture on hot flush and menopause symptoms in breast cancer- a systematic review and meta-analysis," PLoS One, vol. 12, no. 8, Article ID e0180918, 2017.

[13] T. T. Phan, T. T. Ho, H. T. Nguyen, H. Nguyen, T. B. Tran, and S. T. Nguyen, "The prognostic impact of neutrophil to lymphocyte ratio in advanced non-small-cell lung cancer patients treated with EGFR TKI," International Journal of General Medicine, vol. 11, pp. 423-430, 2018.

[14] S. H. Sim, S. H. Beom, Y. O. Ahn et al., "Pretreatment neutrophil-lymphocyte ratio is not a significant prognostic factor in epidermal growth factor receptor-mutant non-small cell lung cancer patients treated with tyrosine kinase inhibitors," Thoracic Cancer, vol. 7, no. 2, pp. 161-166, 2016.

[15] Y. Zhang, Y. C. Feng, H. G Zhu et al., "The peripheral blood neutrophil-to-lymphocyte ratio is a prognostic predictor for survival of EGFR-mutant nonsmall cell lung cancer patients treated with EGFR-TKIs," Medicine, vol. 97, no. 30, Article ID e11648, 2018.

[16] C. Xu, X. Yao, T. Li et al., "Pretreatment neutrophil-tolymphocyte ratio is a predictive biomarker for EGFR TKItreated patients with advanced EGFR- mutant non-small cell lung cancer," Translational Cancer Research, vol. 9, no. 4, pp. 2875-2883, 2020.

[17] G.-N. Lin, J.-W. Peng, P.-P. Liu, D.-Y. Liu, J.-J. Xiao, and X.-Q. Chen, "Elevated neutrophil-to-lymphocyte ratio predicts poor outcome in patients with advanced non-small-cell lung cancer receiving first-line gefitinib or erlotinib treatment," Asia-Pacific Journal of Clinical Oncology, vol. 13, no. 5, pp. e189-e194, 2017.

[18] Y. M. Chen, C. H. Lai, K. M. Rau et al., "Impact of clinical parameters and systemic inflammatory status on epidermal growth factor receptor-mutant non-small cell lung cancer patients readministration with epidermal growth factor receptor tyrosine kinase inhibitors," BMC Cancer, vol. 16, no. 1, p. $868,2016$.

[19] S. H. Saverymuttu, A. M. Peters, A. Keshavarzian, H. J. Reavy, and J. P. Lavender, "The kinetics of ${ }^{111}$ indium distribution following injection of ${ }^{111}$ indium labelled autologous granulocytes in man," British Journal of Haematology, vol. 61, no. 4, pp. 675-685, 1985.

[20] S. K. Wculek and I. Malanchi, "Neutrophils support lung colonization of metastasis-initiating breast cancer cells," Nature, vol. 528, no. 7582, pp. 413-417, 2015.

[21] S. B. Coffelt, M. D. Wellenstein, and K. E. de Visser, "Neutrophils in cancer: neutral no more," Nature Reviews Cancer, vol. 16, no. 7, pp. 431-446, 2016.

[22] A. J. Templeton, M. G. McNamara, B. Šeruga et al., "Prognostic role of neutrophil-to-lymphocyte ratio in solid tumors: 
a systematic review and meta-analysis," JNCI: Journal of the National Cancer Institute, vol. 106, no. 6, Article ID dju124, 2014.

[23] Y. Sawanobori, S. Ueha, M. Kurachi et al., "Chemokinemediated rapid turnover of myeloid-derived suppressor cells in tumor-bearing mice," Blood, vol. 111, no. 12, pp. 5457-5466, 2008.

[24] K. H. Steinbach, P. Schick, F. Trepel et al., "Estimation of kinetic parameters of neutrophilic, eosinophilic, and basophilic granulocytes in human blood," Blut, vol. 39, no. 1, pp. 27-38, 1979.

[25] L. A. Pekarek, B. A. Starr, A. Y. Toledano, and H. Schreiber, "Inhibition of tumor growth by elimination of granulocytes," Journal of Experimental Medicine, vol. 181, no. 1, pp. 435-440, 1995.

[26] C. Neuzillet, A. Casadei Gardini, B. Brieau et al., "Prediction of survival with second-line therapy in biliary tract cancer: actualisation of the AGEO CT2BIL cohort and European multicentre validations," European Journal of Cancer, vol. 111, pp. 94-106, 2019.

[27] O. Brunetti, A. Gnoni, A. Licchetta et al., "Predictive and prognostic factors in HCC patients treated with sorafenib," Medicina, vol. 55, no. 10, p. 707, 2019.

[28] G. P. Dunn, L. J. Old, and R. D. Schreiber, "The three Es of cancer immunoediting," Annual Review of Immunology, vol. 22, no. 1, pp. 329-360, 2004.

[29] P. Bhat, G. Leggatt, N. Waterhouse, and I. H. Frazer, "Interferon- $\gamma$ derived from cytotoxic lymphocytes directly enhances their motility and cytotoxicity," Cell Death \& Disease, vol. 8, no. 6, Article ID e2836, 2017.

[30] P. S. Kim and R. Ahmed, "Features of responding T cells in cancer and chronic infection," Current Opinion in Immunology, vol. 22, no. 2, pp. 223-230, 2010.

[31] J. Kmiecik, A. Poli, N. H. Brons et al., "Elevated $\mathrm{CD}^{+}$and $\mathrm{CD}^{+}$tumor-infiltrating immune cells correlate with prolonged survival in glioblastoma patients despite integrated immunosuppressive mechanisms in the tumor microenvironment and at the systemic level," Journal of Neuroimmunology, vol. 264, no. 1-2, pp. 71-83, 2013.

[32] S. J. Piersma, E. S. Jordanova, M. I. E. van Poelgeest et al., "High number of intraepithelial $\mathrm{CD}^{+}$tumorinfiltrating lymphocytes is associated with the absence of lymph node metastases in patients with large early-stage cervical cancer," Cancer Research, vol. 67, no. 1, pp. 354-361, 2007.

[33] R. E. Tay, E. K. Richardson, and H. C. Toh, "Revisiting the role of CD4(+) T cells in cancer immunotherapy-new insights into old paradigms," Cancer Gene Therapy, 2020.

[34] N. Zhang, J. Jiang, S. Tang, and G. Sun, "Predictive value of neutrophil-lymphocyte ratio and platelet-lymphocyte ratio in non-small cell lung cancer patients treated with immune checkpoint inhibitors: a meta-analysis," International Immunopharmacology, vol. 85, Article ID 106677, 2020. 